\title{
Strong metric dimensions for power graphs of finite groups
}

\author{
Xuanlong $\mathrm{Ma}^{1, *}$ and Liangliang Zhai ${ }^{2}$ \\ ${ }^{1}$ School of Science, Xi'an Shiyou University, Xi'an 710065, China \\ ${ }^{2}$ School of Mathematics, Northwest University, Xi'an 710127, China
}

\begin{abstract}
Let $G$ be a finite group. The order supergraph of $G$ is the graph with vertex set $G$, and two distinct vertices $x, y$ are adjacent if $o(x) \mid o(y)$ or $o(y) \mid o(x)$. The enhanced power graph of $G$ is the graph whose vertex set is $G$, and two distinct vertices are adjacent if they generate a cyclic subgroup. The reduced power graph of $G$ is the graph with vertex set $G$, and two distinct vertices $x, y$ are adjacent if $\langle x\rangle \subset\langle y\rangle$ or $\langle y\rangle \subset\langle x\rangle$. In this paper, we characterize the strong metric dimension of the order supergraph, the enhanced power graph and the reduced power graph of a finite group.
\end{abstract}

Key words: Strong metric dimension; Order supergraph; Enhanced power graph; Reduced power graph; Finite group.

2010 MSC: 05C25; 05C69

\section{Introduction}

All graphs considered in this paper are finite, undirected, with no loops and no multiple edges. Let $\Gamma$ be a graph. The vertex set of $\Gamma$ is denoted by $V(\Gamma)$. Let $x, y, z \in V(\Gamma)$. The distance between $x$ and $y$ in $\Gamma$, denoted by $d(x, y)$, is the length of a shortest path from $x$ to $y$. The diameter of $\Gamma$ is the greatest distance between any two vertices. We say that $z$ strongly resolves $x$ and $y$ if there exists a shortest path from $z$ to $x$ containing $y$, or a shortest path from $z$ to $y$ containing $x$. A subset $S$ of

\footnotetext{
*Corresponding author

E-mail address: xuanlma@mail.bnu.edu.cn (X. Ma), zhailiang111@126.con (L. Zhai)
} 
$V(\Gamma)$ is a strong resolving set of $\Gamma$ if every pair of vertices of $\Gamma$ is strongly resolved by some vertex in $S$. The smallest cardinality of a strong resolving set of $\Gamma$, denoted by $\operatorname{sdim}(\Gamma)$, is called the strong metric dimension of $\Gamma$.

In the 1970s, the metric dimension of a graph was introduced independently by Harary and Melter [18] and Slater [36]. In 2004, Sebö and Tannier [35] introduced the strong metric dimension of a graph and presented some applications of strong resolving sets to combinatorial searching. The problem of computing strong metric dimension is NP-hard [27]. Some theoretical results, computational approaches and recent results on strong metric dimension can be found in [26].

Graphs associated with groups and other algebraic structures have been actively investigated, since they have valuable applications (cf. [21, 25]) and are related to automata theory (cf. $[22,23])$. The undirected power graph $\mathcal{P}(G)$ of a finite group $G$ has vertex set $G$ and two distinct elements are adjacent if one is a power of the other. The concepts of power graph and undirected power graph were first introduced by Kelarev and Quinn [24] and Chakrabarty et al. [9], respectively. The metric dimension and the strong metric dimension of a power graph were studied in [13] and [28], respectively. In recent years, the study of power graphs has been growing, see, for example, $[6-8,30,31]$. Also, see [2] for a survey of results and open problems on power graphs.

Let $G$ be a finite group. The enhanced power graph $\mathcal{P}_{E}(G)$ of $G$ is the graph whose vertex set is $G$, and two distinct vertices are adjacent if they generate a cyclic subgroup of $G$. In order to measure how close the power graph is to the commuting graph, Aalipour et al. [1] introduced the enhanced power graph which lies in between. Ma and She [29] characterized the metric dimension of an enhanced power graph. See $[1,5,10,11,32]$ for some more properties of the enhanced power graph.

The order supergraph $\mathcal{S}(G)$ of $\mathcal{P}(G)$ of $G$ is a graph with vertex set $G$, and two distinct vertices $x, y$ are adjacent if $o(x) \mid o(y)$ or $o(y) \mid o(x)$, where $o(x)$ and $o(y)$ are the orders of $x$ and $y$, respectively. By the definition of an order supergraph, we also call $\mathcal{S}(G)$ as the order supergraph of $G$. In 2017, Hamzeh and Ashrafi [15] called this graph as the main supergraph of $G$ and studied its full automorphism group. Recently, Hamzeh and Ashrafi [16] studied some properties of the order supergraph, and in particular, they showed that $\mathcal{S}(G)=\mathcal{P}(G)$ if and only if $G$ is cyclic. Also, in [17], they investigated Hamiltonianity, Eulerianness and 2-connectedness of this graph.

With an intention to avoid the complexity of edges in the power graphs, Rajkumar and Anitha [33] introduced the reduced power graph $\mathcal{P}_{R}(G)$ of $G$, which is an undirected graph with vertex set $G$, and two distinct vertices $x, y$ are adjacent if $\langle x\rangle \subset\langle y\rangle$ or $\langle y\rangle \subset\langle x\rangle$. In other words, $\mathcal{P}_{R}(G)$ is the subgraph of $\mathcal{P}(G)$ obtained by 
deleting all edges $\{x, y\}$ with $\langle x\rangle=\langle y\rangle$, where $x$ and $y$ are two distinct elements of $G$. In [33], the authors studied the interplay between the algebraic properties of a group and the graph theoretic properties of its reduced power graph. Recently, Anitha and Rajkumar [4] characterized the groups with planar, toroidal and projective planar reduced power graphs. Moreover, see [3, 34] for some more properties of this graph.

According to the definitions as above, for any finite group $G, \mathcal{P}_{R}(G)$ is a spanning subgraph of $\mathcal{P}(G)$, and $\mathcal{P}(G)$ is a spanning subgraph of both $\mathcal{S}(G)$ and $\mathcal{P}_{E}(G)$. In this paper, we characterize the strong metric dimension of the order supergraph, the enhanced power graph and the reduced power graph of a finite group.

\section{Preliminaries}

This section introduces some basic definitions and notations that are used throughout the paper.

Every group considered in this paper is finite. We always use $e$ to denote the identity element of the group under consideration. Let $G$ be a group. The order of an element $x$ of $G$, denoted by $o(x)$, is defined as the cardinality of the cyclic subgroup $\langle x\rangle$. An element of order 2 is called an involution. The exponent of $G$, denoted by $\exp (G)$, is defined as the least common multiple of the orders of all elements of $G$. The set of orders of all elements of $G$ is denoted by $\pi_{e}(G)$. A maximal cyclic subgroup of $G$ is a cyclic subgroup, which is not a proper subgroup of some cyclic subgroup of $G$. The set of all maximal cyclic subgroups of $G$ is denoted by $\mathcal{M}_{G}$. Note that $\left|\mathcal{M}_{G}\right|=1$ if and only if $G$ is cyclic. Denote by $\mathbb{Z}_{n}$ the cyclic group of order $n$.

A finite group is called a $\mathcal{P}$-group [12] if every nontrivial element of the group has prime order. For example, the elementary abelian $p$-group $\mathbb{Z}_{p}^{n}$ is a $\mathcal{P}$-group where $p$ is a prime and $n \geq 1$, and the symmetric group $S_{3}$ on 3 letters is also a $\mathcal{P}$-group. A finite group is called a CP-group [19] if every nontrivial element of the group has prime power order. Clearly, both $p$-groups and $\mathcal{P}$-groups are also CP-groups.

For $n \geq 2$, Johnson [20, pp. 44-45] defined the generalized quaternion group $Q_{4 n}$ of order $4 n$ by the presentation

$$
Q_{4 n}=\left\langle x, y: x^{n}=y^{2}, x^{2 n}=y^{4}=e, y^{-1} x y=x^{-1}\right\rangle .
$$

If $n=2$, then $Q_{8}$ is the usual quaternion group of order 8. Some basic properties of $Q_{4 n}$ can be found in [14]. We remark that $x^{n}$ is the unique involution of $Q_{4 n}$. Also, it is easy to check that

$$
Q_{4 n}=\langle x\rangle \cup\left\{x^{i} y: 1 \leq i \leq 2 n\right\}, o\left(x^{i} y\right)=4 \text { for each } 1 \leq i \leq 2 n
$$


and

$$
\mathcal{M}_{Q_{4 n}}=\left\{\langle x\rangle,\langle x y\rangle, \ldots,\left\langle x^{n} y\right\rangle\right\}, \quad x^{n} \in \bigcap_{M \in \mathcal{M}_{4 n}} M .
$$

Recall now the following elementary result.

Theorem 2.1 ([14, Theorem 5.4.10 (ii)]) Let $p$ be a prime. Then a p-group having a unique subgroup of order $p$ is either cyclic or generalized quaternion.

Let $\Gamma$ be a graph and $x \in V(\Gamma)$. The closed neighborhood of $x$ in $\Gamma$ is

$$
N_{\Gamma}[x]=\{y \in V(\Gamma): d(y, x) \leq 1\}
$$

If the situation is unambiguous, we denote $N_{\Gamma}[x]$ simply by $N[x]$. A subset of $V(\Gamma)$ is called a clique if any two distinct vertices in this subset are adjacent in $\Gamma$. The clique number of $\Gamma$, denoted by $\omega(\Gamma)$, is the maximum cardinality of a clique in $\Gamma$.

For $x, y \in V(\Gamma)$, define a binary relation $x \approx y$ by the rule that $N[x]=N[y]$ in $\Gamma$. Observe that $\approx$ is an equivalence relation over $V(\Gamma)$. Let $U(\Gamma)$ be a complete set of distinct representative elements for this equivalence relation. The reduced graph of $\Gamma$, denoted by $\mathcal{R}_{\Gamma}$, has the vertex set $U(\Gamma)$ and two vertices are adjacent if they are adjacent in $\Gamma$. Notice that for two distinct equivalence classes $\mathcal{C}_{1}$ and $\mathcal{C}_{2}$, if there exist a vertex in $\mathcal{C}_{1}$ and a vertex in $\mathcal{C}_{2}$ which are adjacent in $\Gamma$, then each vertex in $\mathcal{C}_{1}$ and each vertex in $\mathcal{C}_{2}$ are adjacent in $\Gamma$. As a result, $\mathcal{R}_{\Gamma}$ does not depend on the choice of representatives.

Ma et al. [28] characterized the strong metric dimension of a graph with diameter two by the reduced graph of this graph.

Theorem 2.2 ([28, Theorem 2.2]) Let $\Gamma$ be a connected graph with order $n$ and diameter two. Then $\operatorname{sdim}(\Gamma)=n-\omega\left(\mathcal{R}_{\Gamma}\right)$.

For a positive integer $n$, let $n=p_{1}^{r_{1}} p_{2}^{r_{2}} \cdots p_{m}^{r_{m}}$ be its canonical factorization, that is, $p_{1}, p_{2}, \ldots, p_{m}$ are pairwise distinct primes and $r_{i} \geq 1$ for $1 \leq i \leq m$. Denote by $\Omega(n)$ the number of all prime factors of $n$ counted with multiplicity. Namely,

$$
\Omega(n)=\sum_{i=1}^{m} r_{i} .
$$

\section{Order supergraphs of power graphs}

This section characterizes the strong metric dimension of the order supergraph of a group. Our main result is as follows. 
Theorem 3.1 Let $G$ be a group of order $n$. Then

$$
\operatorname{sdim}(\mathcal{S}(G))= \begin{cases}n-1, & \text { if } G \text { is a p-group; } \\ n-\Omega(n), & \text { if } G \text { is a cyclic group and is not a p-group } \\ n-2, & \text { if } G \text { is a CP-group and is not a p-group } \\ n-\lambda_{G}-1, & \text { otherwise, }\end{cases}
$$

where $\lambda_{G}=\max \left\{\Omega(m): m \in \pi_{e}(G)\right.$ and $m$ is not a prime power $\}$.

Note that $\mathcal{S}(G)$ is complete if and only if $G$ is a $p$-group (see also [16, Theorem 2.3]). So, $\operatorname{sdim}(\mathcal{S}(G))=|G|-1$ if and only if $G$ is a $p$-group. As a corollary of Theorem 3.1, we can classify all groups $G$ whose order supergraphs have strong metric dimension $|G|-2$.

Corollary 3.2 Let $G$ be a group of order $n$. Then $\operatorname{sdim}(\mathcal{S}(G))=n-2$ if and only if $G$ is isomorphic to either $\mathbb{Z}_{p q}$ or a CP-group with at least two distinct prime divisors, where $p, q$ are two distinct primes.

By Theorem 3.1 and (2), we determine the strong metric dimension of the order supergraph of a generalized quaternion group.

Corollary 3.3 Let $Q_{4 n}$ be the generalized quaternion group as presented in (1). Then

$$
\operatorname{sdim}\left(\mathcal{S}\left(Q_{4 n}\right)\right)= \begin{cases}4 n-1, & \text { if } n \text { is a power of } 2 \\ 4 n-\Omega(2 n)-1, & \text { otherwise }\end{cases}
$$

In the following, we aim to prove Theorem 3.1. For $x, y \in G$, denote by $\sim$ the equivalence relation defined by $N[x]=N[y]$ in $\mathcal{S}(G)$. As stated above, $\sim$ is an equivalence relation over $G$.

We first prove some results before giving the proof of Theorem 3.1.

Lemma 3.4 Let $G$ be a group such that $|G|$ is divisible by at least two distinct primes. Let $x$ and $y$ be two distinct elements of $G$. Then $x \sim y$ in $\mathcal{S}(G)$ if and only if one of the following occurs:

(i) $o(x)=o(y)$.

(ii) $\{o(x), o(y)\}=\{1, \exp (G)\}$.

(iii) $\{o(x), o(y)\}=\left\{p^{m}, p^{n}\right\}$ and $p^{n} q \notin \pi_{e}(G)$, where $p, q$ are two distinct primes and $m, n$ are two positive integers with $m>n$. 
Proof. By the definition of an order supergraph, the proof of the sufficiency is straightforward. We next prove the necessity. Suppose that $x \sim y$ in $\mathcal{S}(G)$. Assume that $o(x) \neq o(y)$. Suppose that one of $x$ and $y$ is $e$. Without loss of generality, let $x=e$. Then $N[y]=G$. Since $|G|$ is divisible by at least two distinct primes, we have that $o(y)$ is not a prime power. It follows from $N[y]=G$ that $\exp (G) \mid o(y)$. Also, as $o(y)$ divides $\exp (G)$, we actually have that $\exp (G)=o(y)$, as desired.

Suppose, in the following, that $e \notin\{x, y\}$. We claim that if $o(x)$ is not a prime power, then $o(x) \mid o(y)$. In fact, let $q^{t} \mid o(x)$ and $q^{t+1} \nmid o(x)$, where $q$ is a prime. It follows that there exists $a \in G$ such that $o(a)=q^{t}$, and so $a \in N[y]$. Note that $o(x)$ is not a prime power. Let $r \neq q$ be a prime divisor of $o(x)$. It follows that there exists an element of order $r$ such that it belongs to $N[x]=N[y]$, which implies that $o(y)$ is not a power of $q$. As a result, we have $a \neq y$. It follows that $q^{t} \mid o(y)$, and so $o(x) \mid o(y)$. Thus, the claim is valid. We conclude that if $o(x)$ is not a prime power, then $o(y)$ is also not a prime power, it follows from the above claim that $o(y)=o(x)$, a contradiction. So, we may assume that $o(x)=p^{m}$ and $o(y)=p^{n}$ for some prime $p$ and two distinct positive integers $m, n$. Without loss of generality, we may assume that $m>n$. Suppose, to the contrary, that there exists an element $z$ in $G$ such that $o(z)=p^{n} q$ for some prime $q \neq p$. Then $z \in N[y]$, and so $z \in N[x]$. It follows that $p^{m} \mid p^{n} q$, contrary to $m>n$. Thus, the necessity follows.

The following result is immediate by Lemma 3.4.

Corollary 3.5 Let $x, y \in G$ with $\{o(x), o(y)\}=\left\{p^{m}, p^{n}\right\}$, where $p$ is a prime and $m, n$ are positive integers with $m>n$. Then $x \sim y$ if and only if $p^{n} q \notin \pi_{e}(G)$ for any prime $q \neq p$.

For some elements $a_{1}, a_{2}, \ldots, a_{k}$ of $G$, if $o\left(a_{1}\right)\left|o\left(a_{2}\right)\right| \cdots \mid o\left(a_{k}\right)$ and $o\left(a_{i}\right) \neq o\left(a_{j}\right)$ for any two indices $1 \leq i<j \leq k$, then $\left\{a_{1}, a_{2}, \ldots, a_{k}\right\}$ is called a proper order chain of $G$.

Lemma 3.6 If $C$ is a clique of $\mathcal{R}_{\mathcal{S}(G)}$, then $C$ is a proper order chain of $G$.

Proof. Notice that $o(x) \neq o(y)$ for each two distinct $x, y \in C$. We proceed by induction on the size of $C$. If $|C|=2$, the desired result follows. Assume inductively that the result holds for cliques of size $n$. Let $C=\left\{a_{1}, a_{2}, \ldots, a_{n}, a_{n+1}\right\}$. Then, without loss of generality, we may assume that $o\left(a_{1}\right)\left|o\left(a_{2}\right)\right| \cdots \mid o\left(a_{n}\right)$ and $\left\{a_{1}, a_{2}, \ldots, a_{n}\right\}$ is a proper order chain. If $o\left(a_{n+1}\right) \mid o\left(a_{1}\right)$, then the desired result follows. As a result, we may assume that $o\left(a_{1}\right) \mid o\left(a_{n+1}\right)$. Let

$$
k=\max \left\{i: o\left(a_{i}\right) \mid o\left(a_{n+1}\right)\right\} .
$$


If $k=n$, then the required result follows. Otherwise, we must have $o\left(a_{k}\right)\left|o\left(a_{n+1}\right)\right|$ $o\left(a_{k+1}\right)$, as desired.

A graph is called a tree if it is connected and has no cycles. A graph is called a star if it is a tree on $n$ vertices with one vertex having degree $n-1$ and the other $n-1$ vertices having degree 1 .

Theorem 3.7 Let $G$ be a group of order $n$. Then

$\omega\left(\mathcal{R}_{\mathcal{S}(G)}\right)= \begin{cases}1, & \text { if } G \text { is a p-group; } \\ \Omega(n), & \text { if } G \text { is a cyclic group with at least two distinct prime divisor; } \\ 2, & \text { if } G \text { is a CP-group with at least two distinct prime divisors; } \\ \lambda_{G}+1, & \text { otherwise, }\end{cases}$

where $\lambda_{G}=\max \left\{\Omega(m): m \in \pi_{e}(G)\right.$ and $m$ is not a prime power $\}$.

Proof. Note that $\mathcal{S}(G)$ is complete if and only if $G$ is a $p$-group. Thus, if $G$ is a p-group, then $\mathcal{R}_{\mathcal{S}(G)}$ has order 1 , and so $\omega\left(\mathcal{R}_{\mathcal{S}(G)}\right)=1$, as desired. Suppose now that $G$ is a cyclic group with at least two distinct prime divisors. Then it follows from [16, Theorem 2.2] that $\mathcal{S}(G)=\mathcal{P}(G)$. Thus, in view of [28, Theorem 3.1], we have $\omega\left(\mathcal{R}_{\mathcal{S}(G)}\right)=\Omega(n)$, as desired.

Suppose next that $G$ is a CP-group with at least two distinct prime divisors. Then $G$ is non-cyclic. By Lemma 3.4, for distinct $x, y \in G$, we have that $x \sim y$ if and only if $o(x)=p^{m}$ and $o(y)=p^{n}$ where $p$ is a prime. It follows that $\mathcal{R}_{\mathcal{S}(G)}$ is a star, which implies that $\omega\left(\mathcal{R}_{\mathcal{S}(G)}\right)=2$, as desired.

Finally, suppose that $G$ is a non-cyclic group with at least two distinct prime divisors and is not a CP-group. Let $C=\left\{a_{1}, a_{2}, \ldots, a_{t}\right\}$ be a clique of $\mathcal{R}_{\mathcal{S}(G)}$ with $|C|=\omega\left(\mathcal{R}_{\mathcal{S}(G)}\right)$. Then from Lemma 3.6, it follows that $C$ is a proper order chain of $G$. Thus, without loss of generality, we may assume that $o\left(a_{1}\right)\left|o\left(a_{2}\right)\right| \cdots \mid o\left(a_{t}\right)$. Note that

$$
\lambda_{G}=\max \left\{\Omega(m): m \in \pi_{e}(G) \text { and } m \text { is not a prime power }\right\} .
$$

In the following, we first prove

$$
|C| \leq \lambda_{G}+1
$$

If $o\left(a_{t}\right)$ is not a prime power, then it is easy to see that $|C| \leq \lambda_{G}+1$, as desired. Now suppose that $o\left(a_{t}\right)=p^{k}$ for some prime $p$ and positive integer $k$. If $a_{t-1}=e$, then $|C|=2<\lambda_{G}+1$ since $G$ is not a CP-group, as desired. As a result, we may assume that $o\left(a_{t-1}\right)=p^{l}$ for some $1 \leq l<k$. Note that $N\left[a_{t}\right] \neq N\left[a_{t-1}\right]$. By Corollary 3.5, there exists $x \in G$ such that $o(x)=p^{l} q$ for some prime $q \neq p$. 
Therefore, $\left\{a_{1}, a_{2}, \ldots, a_{t-1}, x\right\}$ is also a clique of $\mathcal{R}_{\mathcal{S}(G)}$, which implies that $|C| \leq$ $\Omega(o(x))+1 \leq \lambda_{G}+1$, as desired.

On the other hand, let

$$
m=p_{1}^{r_{1}} p_{2}^{r_{2}} \cdots p_{h}^{r_{h}} \in \pi_{e}(G)
$$

where $h \geq 2, p_{1}, p_{2}, \ldots, p_{h}$ are pairwise distinct primes and $r_{i} \geq 1$ for any $1 \leq i \leq h$. Take $y \in G$ with $o(y)=m$. Now let $T=\left\{e, y_{1}, y_{2}, \cdots, y_{\Omega(m)}\right\}$ be a subset of $\langle y\rangle$ such that

$$
\begin{aligned}
& \left|y_{1}\right|=p_{1},\left|y_{2}\right|=p_{1} p_{2},\left|y_{3}\right|=p_{1}^{2} p_{2},\left|y_{4}\right|=p_{1}^{3} p_{2}, \ldots,\left|y_{r_{1}+1}\right|=p_{1}^{r_{1}} p_{2}, \\
& \left|y_{r_{1}+2}\right|=p_{1}^{r_{1}} p_{2}^{2},\left|y_{r_{1}+3}\right|=p_{1}^{r_{1}} p_{2}^{3}, \ldots,\left|y_{r_{1}+r_{2}}\right|=p_{1}^{r_{1}} p_{2}^{r_{2}}, \\
& \left|y_{r_{1}+r_{2}+1}\right|=p_{1}^{r_{1}} p_{2}^{r_{2}} p_{3},\left|y_{r_{1}+r_{2}+2}\right|=p_{1}^{r_{1}} p_{2}^{r_{2}} p_{3}^{2}, \ldots,\left|y_{r_{1}+r_{2}+r_{3}}\right|=p_{1}^{r_{1}} p_{2}^{r_{2}} p_{3}^{r_{3}}, \\
& \ldots \ldots \\
& \left|y_{r_{1}+r_{2}+\ldots+r_{h-1}+1}\right|=p_{1}^{r_{1}} p_{2}^{r_{2}} \cdots p_{h-1}^{r_{h-1}} p_{h},\left|y_{r_{1}+r_{2}+\ldots+r_{h-1}+2}\right|=p_{1}^{r_{1}} p_{2}^{r_{2}} \cdots p_{h-1}^{r_{h-1}} p_{h}^{2}, \ldots, \\
& \left|y_{r_{1}+r_{2}+\ldots+r_{h-1}+r_{h}-1}\right|=p_{1}^{r_{1}} p_{2}^{r_{2}} \cdots p_{h-1}^{r_{h-1}} p_{h}^{r_{h}-1},\left|y_{\Omega(m)}\right|=m .
\end{aligned}
$$

Note that $G$ is neither a $p$-group nor a cyclic group. By Lemma 3.4, it is easy to see that $T$ is a clique of $\mathcal{R}_{\mathcal{S}(G)}$ with size $\Omega(m)+1$. It follows that $\mathcal{R}_{\mathcal{S}(G)}$ has a clique of size $\lambda_{G}+1$. Now (4) implies that $\omega\left(\mathcal{R}_{\mathcal{S}(G)}\right)=\lambda_{G}+1$, as required.

Theorem 3.1 follows from Theorems 2.2 and 3.7.

\section{Enhanced power graphs}

Panda et al. [32] computed the strong metric dimensions of the enhanced power graphs of some groups, such as, dihedral groups and semi-dihedral groups. In this section, we characterize the strong metric dimension of the enhanced power graph of a group (see Theorem 4.5).

Let $G$ be a group. For any $g \in G$, define

$$
\begin{gathered}
{[g]:=\{x \in G:\langle x\rangle=\langle g\rangle\},} \\
\mathcal{M}_{g}:=\left\{M \in \mathcal{M}_{G}: g \in M\right\},
\end{gathered}
$$

and

$$
\mathcal{C}(g):=\bigcap_{M \in \mathcal{M}_{g}} M \backslash \bigcup_{M \in \mathcal{M}_{G} \backslash \mathcal{M}_{g}} M .
$$

Note that $g \in \mathcal{C}(g)$ and that $\mathcal{C}(e)=\bigcap_{M \in \mathcal{M}_{G}} M$, because $\mathcal{M}_{e}=\mathcal{M}_{G}$. For $x, y \in G$, denote by $\equiv$ the equivalence relation defined by $N[x]=N[y]$ in $\mathcal{P}_{E}(G)$. As stated in 
Section 2 , $\equiv$ is an equivalence relation over $G$. The $\equiv$-class containing the element $x \in G$ is denoted by $\bar{x}$. Let $\bar{G}=\{\bar{x}: x \in G\}$.

Recall that $\mathcal{P}_{E}(G)$ is complete if and only if $G$ is cyclic (see [5, Theorem 2.4]). Thus, if $G$ is a cyclic group, then $\bar{g}=\mathcal{C}(g)=G$ for any $g \in G$, since $\mathcal{M}_{G}=\{G\}$ if and only if $G$ is cyclic. Now in view of [29, Proposition 2.3], we have the following result, which characterizes every $\equiv$-class.

Lemma 4.1 For every $g \in G$, we have $\bar{g}=\mathcal{C}(g)$. In particular, $[g] \subseteq \bar{g}$.

Lemma 4.2 A maximal clique of $\mathcal{R}_{\mathcal{P}_{E}(G)}$ is a subset of some maximal cyclic subgroup of $G$.

Proof. By the definition of $\mathcal{R}_{\mathcal{P}_{E}(G)}$, it is easy to see that A maximal clique in $\mathcal{R}_{\mathcal{P}_{E}(G)}$ is also a clique in $\mathcal{P}_{E}(G)$. Now [1, Lemma 33] implies that a maximal clique in the enhanced power graph is a cyclic subgroup, so a maximal clique of $\mathcal{R}_{\mathcal{P}_{E}(G)}$ is a subset of some maximal cyclic subgroup of $G$.

Lemma 4.3 If $\left\{x_{1}, x_{2}, \ldots, x_{t}\right\}$ is a maximal clique of $\mathcal{R}_{\mathcal{P}_{E}(G)}$, then $\bigcup_{i=1}^{t} \overline{x_{i}}$ is a maximal cyclic subgroup of $G$.

Proof. By Lemma 4.2, there exists $\langle x\rangle \in \mathcal{M}_{G}$ such that $\left\{x_{1}, x_{2}, \ldots, x_{t}\right\} \subseteq\langle x\rangle$. Also, note that for any $1 \leq i \leq t$, we have $\langle x\rangle \in \mathcal{M}_{x_{i}}$. It follows from Lemma 4.1 and (5) that $\overline{x_{i}} \subseteq\langle x\rangle$, and so $\bigcup_{i=1}^{t} \overline{x_{i}} \subseteq\langle x\rangle$. It suffices to prove that $\langle x\rangle \subseteq \bigcup_{i=1}^{t} \overline{x_{i}}$. Suppose, to the contrary, that there exists $y \in\langle x\rangle$ such that $y \notin \bigcup_{i=1}^{t} \overline{x_{i}}$. Then, similarly, we can deduce that $\bar{y} \subseteq\langle x\rangle$. Note that $y$ is adjacent to $x_{i}$ in $\mathcal{P}_{E}(G)$. We then have that $\left\{x_{1}, x_{2}, \ldots, x_{t}, y\right\}$ is a clique of $\mathcal{R}_{\mathcal{P}_{E}(G)}$, this contradicts our hypothesis that $\left\{x_{1}, x_{2}, \ldots, x_{t}\right\}$ is a maximal clique of $\mathcal{R}_{\mathcal{P}_{E}(G)}$.

Lemma 4.4 Let $x, y \in G$. Then

(i) $N_{\mathcal{P}_{E}(G)}[x]=\bigcup_{M \in \mathcal{M}_{x}} M$.

(ii) $x \equiv y$ if and only if $\mathcal{M}_{x}=\mathcal{M}_{y}$.

Proof. (i) Taking $w \in N_{\mathcal{P}_{E}(G)}[x]$, we have that $\langle x, w\rangle$ is cyclic, and so there exists a maximal cyclic subgroup $M$ such that $\langle x, w\rangle \subseteq M$. As a result, $M \in \mathcal{M}_{x}$, which implies that $w \in M \subseteq \bigcup_{M \in \mathcal{M}_{x}} M$. So, $N_{\mathcal{P}_{E}(G)}[x] \subseteq \bigcup_{M \in \mathcal{M}_{x}} M$. On the other hand, for any $z \in \bigcup_{M \in \mathcal{M}_{x}} M$, we have $z \in N$ for some $N \in \mathcal{M}_{x}$. It follows that $\langle x, z\rangle$ is cyclic, and hence $z \in N_{\mathcal{P}_{E}(G)}[x]$. Namely, $\bigcup_{M \in \mathcal{M}_{x}} M \subseteq N_{\mathcal{P}_{E}(G)}[x]$, as desired.

(ii) If $\mathcal{M}_{x}=\mathcal{M}_{y}$, then (i) implies $N_{\mathcal{P}_{E}(G)}[x]=N_{\mathcal{P}_{E}(G)}[y]$, and so $x \equiv y$, as desired. For the converse, suppose that $x \equiv y$. Let $\langle g\rangle \in \mathcal{M}_{x}$. Then $g \in N_{\mathcal{P}_{E}(G)}[x]$ by (i). 
Since $N_{\mathcal{P}_{E}(G)}[x]=N_{\mathcal{P}_{E}(G)}[y]$, we have that $\langle g, y\rangle$ is cyclic. Now from $\langle g\rangle \in \mathcal{M}_{G}$, it follows that $\langle g, y\rangle=\langle g\rangle$, so $\langle g\rangle \in \mathcal{M}_{y}$. As a result, $\mathcal{M}_{x} \subseteq \mathcal{M}_{y}$. Similarly, we also can deduce $\mathcal{M}_{y} \subseteq \mathcal{M}_{x}$.

Combining Lemmas 4.3, 4.4 and Theorem 2.2, we obtain the main result of this section.

Theorem 4.5 Let $G$ be a group of order $n$. Then

$$
\begin{aligned}
\operatorname{sdim}\left(\mathcal{P}_{E}(G)\right) & =n-\max \left\{|\bar{M}|: M \in \mathcal{M}_{G}\right\} \\
& =n-\max \left\{|S|: S \subseteq M \in \mathcal{M}_{G} \text { and for any } x, y \in S, \mathcal{M}_{x} \neq \mathcal{M}_{y}\right\} .
\end{aligned}
$$

The following result is immediate by Theorem 4.5.

Corollary 4.6 Let $G$ be a group of order $n$. Then

(i) $\operatorname{sdim}\left(\mathcal{P}_{E}(G)\right)=n-1$ if and only if $G$ is cyclic.

(ii) If $G$ is a non-cyclic $\mathcal{P}$-group, then $\operatorname{sdim}\left(\mathcal{P}_{E}(G)\right)=n-2$.

By Theorem 4.5, (2) and (3), we determine the strong metric dimension of the enhanced power graph of a generalized quaternion group.

Corollary 4.7 Let $Q_{4 n}$ be the generalized quaternion group as presented in (1). Then $\operatorname{sdim}\left(\mathcal{P}_{E}\left(Q_{4 n}\right)\right)=4 n-2$.

As an application of Theorem 4.5, we determine the strong metric dimension of the enhanced power graph of an abelian $p$-group.

Proposition 4.8 Let $G$ be a non-cyclic abelian p-group with order $n$ and exponent $p^{m}$. Then $\operatorname{sdim}\left(\mathcal{P}_{E}(G)\right)=n-m-1$.

Proof. Note that $G$ is non-cyclic. We may assume that $G=A \times B$ where $A$ is an abelian $p$-group and $B=\langle b\rangle$ with $o(b)=p^{m}$. Then $\langle(e, b)\rangle \cong B$ is a maximal cyclic subgroup of order $p^{m}$. Clearly,

$$
\langle(e, b)\rangle=\left[\left(e, b^{p^{m}}\right)\right] \cup\left[\left(e, b^{p^{m-1}}\right)\right] \cup\left[\left(e, b^{p^{m-2}}\right)\right] \cup \cdots \cup\left[\left(e, b^{p}\right)\right] \cup\left[\left(e, b^{p^{0}}\right)\right] .
$$

Let $a \in A$ with order $p$. In the following, we prove that for any two $0 \leq i<j \leq m$,

$$
\overline{\left(e, b^{p^{i}}\right)} \neq \overline{\left(e, b^{p^{j}}\right)} .
$$

Note that $i \leq j-1 \leq m-1$. Now $o\left(\left(a, b^{p^{j-1}}\right)\right)=p^{m-j+1}$ and $\left(e, b^{p^{j}}\right) \in\left\langle\left(a, b^{p^{j-1}}\right)\right\rangle$. Let $M \in \mathcal{M}_{G}$ with $\left\langle\left(a, b^{p^{-1}}\right)\right\rangle \subseteq M$. Then $\left.M \in \mathcal{M}_{\left(e, b^{p}\right.}\right)$. Assume, to the contrary, 
that $\left(e, b^{p^{i}}\right) \in M$. Note that $o\left(\left(e, b^{p^{i}}\right)\right)=p^{m-i}$ and $M$ is a cyclic $p$-group. If $m-i>$ $m-j+1$, then $\left\langle\left(a, b^{p^{j-1}}\right)\right\rangle \subseteq\left\langle\left(e, b^{p^{i}}\right)\right\rangle$, a contradiction. Since $0 \leq i<j \leq m$, it follows that $m-i=m-j+1$. This means that the order of $\left\langle\left(a, b^{p^{j-1}}\right)\right\rangle$ is equal to the order of $\left\langle\left(e, b^{p^{i}}\right)\right\rangle$. Since $\left(e, b^{p^{i}}\right) \in M$ and $\left(a, b^{p^{j-1}}\right) \in M$, we obtain a contradiction as $\left\langle\left(a, b^{p^{j-1}}\right)\right\rangle \neq\left\langle\left(e, b^{p^{i}}\right)\right\rangle$.

We conclude $M \notin \mathcal{M}_{\left(e, b^{p^{i}}\right)}$, and so $\mathcal{M}_{\left(e, b^{p^{i}}\right)} \neq \mathcal{M}_{\left(e, b^{p^{j}}\right)}$. Now Lemma 4.4(ii) implies that (7) is valid. It follows from (6) and Lemma 4.1 that $\overline{\langle(e, b)\rangle}=m+1$. Also, note that the fact that a maximal cyclic subgroup of order $p^{t}$ has at most $t+1 \equiv$-classes. Since $G$ has exponent $p^{m}$, we have $\operatorname{sdim}\left(\mathcal{P}_{E}(G)\right)=n-m-1$ by Theorem 4.5.

\section{$5 \quad$ Reduced power graphs}

In this section, we characterize the strong metric dimension of the reduced power graph of a group. Our main result is the following theorem.

Theorem 5.1 Let $G$ be a group of order $n$. Then

$$
\operatorname{sdim}\left(\mathcal{P}_{R}(G)\right)= \begin{cases}2^{k}-k, & \text { if } G \cong \mathbb{Z}_{2^{k}}, \text { where } k \geq 1 \\ 2^{t+2}-t-1, & \text { if } G \cong Q_{4 \cdot 2^{t}}, \text { where } t \geq 1 \\ n-\max \left\{\Omega(m): m \in \pi_{e}(G)\right\}-1, & \text { otherwise. }\end{cases}
$$

In the following, we prove some results before giving the proof of Theorem 5.1.

Lemma 5.2 Let $x$ and $y$ be two distinct elements of $G$. Then $N[x]=N[y]$ in $\mathcal{P}_{R}(G)$ if and only if $G$ is isomorphic to either $\mathbb{Z}_{2^{m}}$ or $Q_{4 \cdot 2^{m}}$ where $m$ is a positive integer, and $\{x, y\}=\{e, a\}$ where $a$ is the unique involution of $G$.

Proof. If $G \cong \mathbb{Z}_{2^{m}}$, clearly, $N[e]=N[a]=G$ where $a$ is the unique involution of $G$, as desired. If $G \cong Q_{4 \cdot 2^{m}}$, it follows from (3) that $N[e]=N[a]=G$, where $a$ is the unique involution of $G$, as desired. Thus, the sufficiency follows.

We next prove the necessity. Let $x$ and $y$ be distinct elements of $G$ and assume that $N[x]=N[y]$ in the graph $\mathcal{P}_{R}(G)$. Since $y^{-1} \in N[x]=N[y]$, it follows that $y=y^{-1}$. Similarly $x=x^{-1}$. As $x$ and $y$ are adjacent in $\mathcal{P}_{R}(G)$, we must have that $\{x, y\}=\{e, a\}$, where $a$ is an involution. Observe that $N[a]=N[e]=G$. From this observation, we deduce that $G$ must be a 2 -group and that $a$ must be the unique involution of $G$. Now in view of Theorem 2.1, we have that $G$ is isomorphic to either $\mathbb{Z}_{2^{m}}$ or $Q_{4 \cdot 2^{m}}$, as wanted.

Lemma 5.3 If $\mathcal{C}$ is a clique in $\mathcal{P}_{R}(G)$, then $\langle\mathcal{C}\rangle$ is cyclic. 
Proof. We shall use induction on $|\mathcal{C}|$. The result is trivial for $|\mathcal{C}|=2$ and so assume that $|\mathcal{C}|>2$. Fix $x \in \mathcal{C}$. If $\langle y\rangle \subset\langle x\rangle$ for every $y \in \mathcal{C} \backslash\{x\}$, then $\langle\mathcal{C}\rangle \subseteq\langle x\rangle$ and so $\langle\mathcal{C}\rangle$ is cyclic. If $\langle x\rangle \subset\langle y\rangle$ for some $y \in \mathcal{C} \backslash\{x\}$, then $\langle\mathcal{C}\rangle \subseteq\langle\mathcal{C} \backslash\{x\}\rangle$. The subgroup $\langle\mathcal{C} \backslash\{x\}\rangle$ is cyclic by our induction hypothesis, and so it follows that $\langle\mathcal{C}\rangle$ is cyclic in this case too. The induction argument goes through.

The following result determines the clique number of a reduced power graph, which also was proved in [34] by an alternative method.

Lemma 5.4 Let $G$ be a group. Then $\omega\left(\mathcal{P}_{R}(G)\right)=\max \left\{\Omega(m): m \in \pi_{e}(G)\right\}+1$.

Proof. Let $k=\max \left\{\Omega(m): m \in \pi_{e}(G)\right\}+1$ and let $\left\{x_{1}, x_{2}, \ldots, x_{t}\right\}$ be a clique of $\mathcal{P}_{R}(G)$ with size $\omega\left(\mathcal{P}_{R}(G)\right)$. It suffices to prove $t=k$. By Lemma 5.3, we have that $\left\{x_{1}, x_{2}, \ldots, x_{t}\right\} \subseteq\langle x\rangle$ for some $x \in G$. Now let $o(x)=m$. Note that for each two $1 \leq i<j \leq t, o\left(x_{i}\right) \neq o\left(x_{j}\right)$, and $o\left(x_{i}\right) \mid o\left(x_{j}\right)$ or $o\left(x_{j}\right) \mid o\left(x_{i}\right)$. Also, $\left\{x_{1}, x_{2}, \ldots, x_{t}\right\}$ must be a clique of $\mathcal{P}_{R}(\langle x\rangle)$ with size $\omega\left(\mathcal{P}_{R}(\langle x\rangle)\right)$. We deduce that $t=\Omega(m)+1$, and so $t \leq k$.

On the other hand, let $n \in \pi_{e}(G)$ with $k=\Omega(n)+1$ and let

$$
n=p_{1}^{r_{1}} p_{2}^{r_{2}} \cdots p_{m}^{r_{m}},
$$

where $p_{1}, p_{2}, \ldots, p_{m}$ are pairwise distinct primes and $r_{i} \geq 1$ for any $1 \leq i \leq m$. Take $a \in G$ with $o(a)=n$. Let $T=\left\{e, a_{1}, a_{2}, \cdots, a_{\Omega(n)}\right\}$ be a subset of $\langle a\rangle$ such that

$$
\begin{aligned}
& \left|a_{1}\right|=p_{m},\left|a_{2}\right|=p_{m}^{2}, \ldots,\left|a_{r_{m}}\right|=p_{m}^{r_{m}}, \\
& \left|a_{r_{m}+1}\right|=p_{m-1} p_{m}^{r_{m}}, \ldots,\left|a_{r_{m}+r_{m-1}}\right|=p_{m-1}^{r_{m-1}} p_{m}^{r_{m}}, \\
& \left|a_{r_{m}+r_{m-1}+1}\right|=p_{m-2} p_{m-1}^{r_{m-1}} p_{m}^{r_{m}}, \ldots,\left|a_{\Omega(n)-1}\right|=p_{1}^{r_{1}-1} p_{2}^{r_{2}} \cdots p_{m}^{r_{m}},\left|a_{\Omega(n)}\right|=p_{1}^{r_{1}} p_{2}^{r_{2}} \cdots p_{m}^{r_{m}} .
\end{aligned}
$$

Now it is easy to see that $T$ is a clique in $\mathcal{P}_{R}(G)$ with size $\Omega(n)+1$, and so $k \leq t$.

Lemma 5.5 Let $G$ be a group. Then

$$
\omega\left(\mathcal{R}_{\mathcal{P}_{R}(G)}\right)= \begin{cases}k, & \text { if } G \cong \mathbb{Z}_{2^{k}}, \text { where } k \geq 1 ; \\ t+1, & \text { if } G \cong Q_{4 \cdot 2^{t}}, \text { where } t \geq 1 ; \\ \max \left\{\Omega(m): m \in \pi_{e}(G)\right\}+1, & \text { otherwise. }\end{cases}
$$

Proof. Suppose that $G \cong \mathbb{Z}_{2^{k}}$ or $Q_{4 \cdot 2^{t}}$, where $k, t \geq 1$. Lemma 5.2 implies that $\mathcal{R}_{\mathcal{P}_{R}(G)}$ is isomorphic to the subgraph of $\mathcal{P}_{R}(G)$ obtained by deleting the vertex $e$ from $\mathcal{P}_{R}(G)$. Note that $e$ is adjacent to every non-identity element of $G$ in $\mathcal{P}_{R}(G)$. As a result, we have that $\omega\left(\mathcal{R}_{\mathcal{P}_{R}(G)}\right)=\max \left\{\Omega(m): m \in \pi_{e}(G)\right\}$. If $G \cong \mathbb{Z}_{2^{k}}$, then $\max \left\{\Omega(m): m \in \pi_{e}(G)\right\}=\Omega\left(2^{k}\right)=k$, as desired. Also, if $G \cong Q_{4 \cdot 2^{t}}$, then by (2), we deduce $\max \left\{\Omega(m): m \in \pi_{e}(G)\right\}=\Omega\left(2^{t+1}\right)=t+1$, as desired. 
Suppose that $G$ is neither $\mathbb{Z}_{2^{k}}$ nor $Q_{4 \cdot 2^{t}}$. By Lemma 5.2, we have that $\mathcal{R}_{\mathcal{P}_{R}(G)}$ is equal to $\mathcal{P}_{R}(G)$, and so the desired result follows from Lemma 5.4.

Remark that $\mathcal{P}_{R}(G)$ is complete if and only if $G \cong \mathbb{Z}_{2}$. Thus, if $G ¥ \mathbb{Z}_{2}$, then $\mathcal{P}_{R}(G)$ has diameter two. Note that the strong metric dimension of a complete graph of order $n$ is $n-1$. Thus, combining Theorem 2.2 and Lemma 5.5, we complete the proof of Theorem 5.1.

By Theorem 5.1 and (2), we determine the strong metric dimension of the reduced power graph of a generalized quaternion group.

Corollary 5.6 Let $Q_{4 n}$ be the generalized quaternion group as presented in (1). Then

$$
\operatorname{sdim}\left(\mathcal{P}_{R}\left(Q_{4 n}\right)\right)= \begin{cases}2^{t+2}-t-1, & \text { if } n=2^{t} \text { for some } t \geq 1 ; \\ 4 n-\Omega(2 n)-1, & \text { otherwise }\end{cases}
$$

Clearly, for a group $G$ of order $n, \operatorname{sdim}\left(\mathcal{P}_{R}(G)\right)=n-1$ if and only if $G$ is isomorphic to the cyclic group of order 2. As a direct application of Theorem 5.1, we conclude the paper by characterizing all groups $G$ whose reduced power graphs have strong metric dimension $n-2$.

Corollary 5.7 The following are equivalent for a group $G$ of order $n$ :

(a) $\operatorname{sdim}\left(\mathcal{P}_{R}(G)\right)=n-2$;

(b) $\mathcal{R}_{\mathcal{P}_{R}(G)}$ is a star;

(c) $G$ is isomorphic to $\mathbb{Z}_{4}, Q_{8}$ or a $\mathcal{P}$-group.

Acknowledgements We are grateful to the anonymous referee for careful reading and helpful comments.

This research was supported by the National Natural Science Foundation of China (Grant Nos. 11801441 and 61976244), the Natural Science Basic Research Program of Shaanxi (Program No. 2020JQ-761), and the Young Talent fund of University Association for Science and Technology in Shaanxi, China (Grant No. 20190507).

\section{References}

[1] G. Aalipour, S. Akbari, P.J. Cameron, R. Nikandish, F. Shaveisi, On the structure of the power graph and the enhanced power graph of a group, Electron. J. Combin. 24 (2017) \#P3.16

[2] J. Abawajy, A. Kelarev, M. Chowdhury, Power graphs: A survey, Electron. J. Graph Theory Appl. 1 (2013) 125-147 
[3] T. Anitha, R. Rajkumar, On the power graph and the reduced power graph of a finite group, Commun. Algebra 47 (2019) 3329-3339

[4] T. Anitha, R. Rajkumar, Characterization of groups with planar, toroidal or projective planar (proper) reduced power graphs, J. Algebra Appl., to appear

[5] S. Bera, A.K. Bhuniya, On enhanced power graphs of finite groups, J. Algebra Appl. 17 (2018) 1850146, 8 pp

[6] D. Bubboloni, M.A. Iranmanesh, S.M. Shaker, On some graphs associated with the finite alternating groups, Commun. Algebra 45 (2017) 5355-5373

[7] P.J. Cameron, S. Ghosh, The power graph of a finite group, Discrete Math. 311 (2011) 1220-1222

[8] P.J. Cameron, H. Guerra, Š. Jurina, The power graph of a torsion-free group, J. Algebr. Comb. 49 (2019) 83-98

[9] I. Chakrabarty, S. Ghosh, M.K. Sen, Undirected power graphs of semigroups, Semigroup Forum 78 (2009) 410-426

[10] D.G. Costanzo, M.L. Lewis, S. Schmidt, E. Tsegaye, G. Udell, The cyclic graph (deleted enhanced power graph) of a direct product, Involve 24 (2020) 167-179

[11] D.G. Costanzo, M.L. Lewis, S. Schmidt, E. Tsegaye, G. Udell, The cyclic graph of a Z-group, Bull. Aust. Math. Soc., Published online (2020), DOI:10.1017/s0004972720001318

[12] M. Deaconescu, Classification of finite groups with all elements of prime order, Proc. Amer. Math. Soc. 106 (1989) 625-629

[13] M. Feng, X. Ma, K. Wang, The structure and metric dimension of the power graph of a finite group, Eur. J. Combin. 43 (2015) 82-97

[14] D. Gorenstein, Finite Groups, Chelsea Publishing Co., New York, 1980

[15] A. Hamzeh, A.R. Ashrafi, Automorphism group of supergraphs of the power graph of a finite group, Eur. J. Combin. 60 (2017) 82-88

[16] A. Hamzeh, A.R. Ashrafi, The order supergraph of the power graph of a finite group, Turk. J. Math. 42 (2018) 1978-1989

[17] A. Hamzeh, A.R. Ashrafi, Some remarks on the order supergraph of the power graph of a finite group, Int. Electron. J. Algebra 26 (2019) 1-12 
[18] F. Harary, R. A. Melter, On the metric dimension of a graph, Ars Combin. 2 (1976) 191-195

[19] G. Higman, Finite groups in which every element has prime power order, J. London Math. Soc. 32 (1957) 335-342

[20] D.L. Johnson, Topics in the Theory of Group Presentations, London Math. Soc. Lecture Note Ser., vol. 42, Cambridge University Press, Cambridge-New York, 1980

[21] A.V. Kelarev, Ring Constructions and Applications, World Scientific, River Edge, NJ, 2002

[22] A.V. Kelarev, Graph Algebras and Automata, Marcel Dekker, New York, 2003

[23] A.V. Kelarev, Labelled Cayley graphs and minimal automata, Australas. J. Combin. 30 (2004) 95-101

[24] A.V. Kelarev, S.J. Quinn, A combinatorial property and power graphs of groups, Contrib. General Algebra 12 (2000) 229-235

[25] A.V. Kelarev, J. Ryan, J. Yearwood, Cayley graphs as classifiers for data mining: The influence of asymmetries, Discret. Math. 309 (2009) 5360-5369

[26] J. Kratica, V. Kovačević-Vujčić, M. Čangalović, N. Mladenović, Strong metric dimension: a survey, Yugosl. J. Oper. Res. 24 (2014) 187-198

[27] D. Kuziak, I.G. Yero, J.A.Rodríguez-Velázquez, On the strong metric dimension of the strong products of graphs, Open Math. 13 (2015) 64-74

[28] X, Ma, M. Feng, K. Wang, The strong metric dimension of the power graph of a finite group, Discrete Appl. Math. 239 (2018) 159-164

[29] X, Ma, Y. She, The metric dimension of the enhanced power graph of a finite group, J. Algebra Appl. 19 (2020) 2050020, 14 pp

[30] A.R. Moghaddamfar, S. Rahbariyan, W.J. Shi, Certain properties of the power graph associated with a finite group, J. Algebra Appl. 13 (2014) 1450040, 18 pp

[31] X. Ma, G.L. Walls, K. Wang, Power graphs of (non)orientable genus two, Commun. Algebra 47 (2019) 276-288

[32] R. P. Panda, S. Dalal, J. Kumar, On the enhanced power graph of a finite group, Commun. Algebra 49 (2021) 1697-1716 
[33] R. Rajkumar, T. Anitha, Reduced power graph of a group, Electron. Notes Discrete Math. 63 (2017) 69-76

[34] R. Rajkumar, T. Anitha, Some results on the reduced power graph of a group, Southeast Asian Bull. Math. 45 (2021) 241-262

[35] A. Sebő, E. Tannier, On metric generators of graphs, Math. Oper. Res. 29 (2004) 383-393

[36] P.J. Slater, Leaves of trees, Congr. Numer. 14 (1975) 549-559 